\title{
Modeling of Intermesh Schedule for Strong Heavy Plate Roller Straightener and Finite Element Method Modification
}

\author{
Xu Meng ${ }^{1,2}$, Sun Dengyue ${ }^{1, a}$, Xu Shimin $^{1}$, Chen Peng $^{1}$ and Sun Hao ${ }^{1}$ \\ ${ }^{1}$ National Cold Rolled Strip Equipment and Process Engineering Technology Research Center, Yan Shan University ,066004 Hebei ,China \\ ${ }^{2}$ Capital Spaceflight Machinery Company, 100076 Beijing, China
}

\begin{abstract}
Regarding straightening unit as the research object, considered the different of roller spacing, a mathematical model of intermesh schedule suitable to the 2800 seven-roller strong heavy plate straightening machine was deduced by the geometric method. According to the mathematical model, the intermesh schedules of several specifications of plate were calculated, and the finite element model of straightening process was established in the finite element analysis software Abaqus. By analysing, it was found that those plates after straightening cannot meet the requirement of flatness, due to the existence of the work hardening. The bending deflection of the last straightening unit was modified and the new calculation formula of intermesh schedule was obtained. The values of the modified coefficients were determined by the finite element method. The intermesh schedules of other specifications of plate were calculated by the modified calculation formula and then verified it in Abaqus. The simulation results showed that those plates after straightening meet the requirement of flatness. So, the research results provide a theoretical basis for development of a new plate straightening machine and formulation of intermesh schedule.
\end{abstract}

\section{General instructions}

At present, the straightening intermesh schedule of strip and medium thin plate is studied more [1], but the heavy plate more than $50 \mathrm{~mm}$ is usually straightened by the less efficient pressure straightening machine. And the algorithm of intermesh schedule for some thick plate roller straightening machine ignore the different of roller spacing, and it is inaccuracy. To obtain the straightening intermesh schedule meeting the requirements the repeated adjusting with experience is needed [2].

In order to improve the speed of formulating intermesh schedule and the straightening work efficiency, for the 2800 seven-roller strong heavy plate straightening machine designed by Yanshan University, a mathematical model of intermesh schedule is deduced. Introduced modified coefficient $X$, through the simulation of the finite element software Abaqus, the effect of plate thickness and yield strength on straightening result is studied, and the value curves of thickness effect coefficient and strength effect coefficient are fitted, then the value of modified coefficient $X$ is determined and verified by the finite element method (FEM). The modified calculation formula Improve the accuracy of formulate intermesh schedule and reduce the number of adjustment.

\section{Deduction of intermesh schedule mathematical model}

\footnotetext{
a Sun Dengyue: sdy@ysu.edu.cn
}

\subsection{Brief Introduction of 2800 Seven-Roller Strong Heavy Plate Straightening Machine}

The distribution of upper three and under four is adopted by the 2800 seven roller plate straightening machine, as shown in Figure 1. The upper rollers can be adjusted vertically or inclinedly as a whole, and the import and export guide rollers which can be adjusted independently are set in the under roller system, and the intermediate two rollers are fixed. The straightening process is reversible, and it's each work roller is vertically supported by three back rollers. Work roller diameter $D_{w}=480 \mathrm{~mm}$, back roller diameter $D_{b}=500 \mathrm{~mm}$, roller spacing of under edge guide rollers $t_{l}=600 \mathrm{~mm}$, roller spacing of middle rollers $t_{2}=520 \mathrm{~mm}$; the maximum total straightening force $\sum P=30000 \mathrm{kN}$, the maximum straightening power $N=1000 \mathrm{~kW}$; straightening range: the thickness is $30-75 \mathrm{~mm}$, the maximum width is 2650 $\mathrm{mm}$, the maximum yield strength is $700 \mathrm{MPa}$.

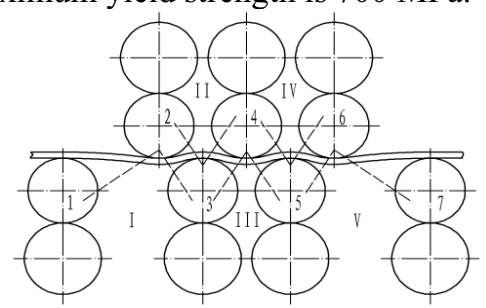

Figure 1. Distribution of roll system and straightening unit 


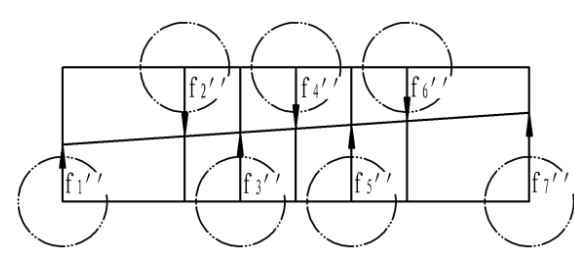

Figure 2. Diagram of the roll system adjustment

\subsection{The Modeling Process}

There are 5 straightening units in the roll system, whose distribution is shown in Figure 1. Due to the adopting of large deformation straightening scheme, the amount of intermesh is large at import terminal, and the plate is not so easy to enter the roll system. So the intermesh amount of the first straightening unit is decreased, just eliminating the inconsistency of plate curvature in a certain extent, so the second straightening unit is the first straightening unit of large deformation straightening scheme in the real sense.

Assuming the 2\#,4\# and 6\# rollers were fixed, and the $3 \#$ and $5 \#$ rollers could move vertically. Let the reverse bending curvature of $3 \#$ roller is equal to the elastic back curvature $1 / \rho_{3}$ which is needed to straighten the original curvature $-1 / r_{0}$, according to the iterative formula [3]:

$$
\frac{1}{\rho_{3}}=\left[3-\left(\frac{\frac{1}{\rho_{w}}}{\frac{1}{r_{0}}+\frac{1}{\rho_{3}}}\right)^{2}\right] \frac{1-\eta}{2 \rho_{w}}+\eta\left(\frac{1}{r_{0}}+\frac{1}{\rho_{3}}\right)
$$

Where: $\eta$ - the material hardening coefficient;

$1 / \rho_{w}$ - - the yield curvature, whose value is $2 \sigma$ ${ }_{s} /(E h)$, the $\sigma_{s}$ is yield strength of material and the $E$ is elastic modulus of material

The elastic back curvature $1 / \rho_{3}$ is gotten through iteration.

According to the "virtual fulcrum" theory [5], the zero bending moment point of intermediate roll approximate to the midpoint of the adjacent rollers. Considering the large deformation scheme, the anti bending deflection of 3 \# roller in the second straightening unit is:

$$
f_{3}{ }^{\prime \prime}=\xi_{3} f_{3}{ }^{\prime \prime}=\xi_{3} \frac{t_{2}{ }^{2}}{48 \rho_{3}}
$$

Where: $\xi_{3}-$ the coefficient considering large deformation degree

Let the anti bending deflection of $5 \#$ roller equal to the maximum value of elastic bending deflection:

$$
f_{5}^{\prime \prime}=\frac{t_{2}^{2}}{48 \rho_{w}}
$$

For the first and fifth straightening unit, due to the diffidence of roller spacing, the zero bending moment point is no longer at the midpoint of straightening unit. The anti bending deflection of $2 \#$ and $6 \#$ roller respectively is:

$$
f_{2}^{\prime \prime}=\frac{t_{2}}{24}\left(2 t_{1}-t_{2}\right) \frac{1}{\rho_{2}}
$$

$$
f_{6}{ }^{\prime \prime}=\frac{t_{2}}{24}\left(2 t_{1}-t_{2}\right) \frac{1}{\rho_{6}}
$$

Let the reverse bending curvature of $2 \#$ roller equal to the the elastic back curvature of $3 \#$ roller $1 / \rho_{3}$, and let the reverse bending curvature of $6 \#$ roller equal to the the yield curvature $1 / \rho_{w}$.

According to geometrical relationship the adjustment amount of each roller is deduced, ignoring the displacement of the upper rollers in the horizontal direction because of the overall inclination adjustment of upper roller system.

For the movable roller, its adjustment amount is equal to the sum of its reverse bending deflection and a half of the sum of reverse bending deflections of its adjacent rollers.

For the fixed rollers, there is no need to get the adjustment amount, but the reverse bending deflection should be calculated, whose value is the average value of its adjacent two rollers.

Assuming the upper rollers were fixed, so the adjustment amount of $3 \#$ and $5 \#$ roller respectively is:

$$
\begin{aligned}
& \Delta h_{3}=f_{3} "+\frac{1}{2}\left(f_{2} "+f_{4} "\right) \\
& \Delta h_{5}=f_{5} "+\frac{1}{2}\left(f_{4} "+f_{6} "\right)
\end{aligned}
$$

According to geometrical relationship of relative motion shown in Figure 2, adjustment amount of 4\# roller is equal to the average value of adjustment amount of $3 \#$ roller and $5 \#$ roller. That is:

$$
\Delta h_{4}=\frac{1}{2}\left(\Delta h_{3}+\Delta h_{5}\right)
$$

And because the upper roller system can be adjusted overall in practice, the reverse bending deflection of $4 \#$ roller is also calculated:

$$
\Delta h_{4}=f_{4}{ }^{\prime \prime}+\frac{1}{2}\left(f_{3} "+f_{5} "\right)
$$

So reverse bending deflection of $4 \#$ roller is obtained according to the equation (6)-(9):

$$
f_{4}{ }^{\prime \prime}=\frac{1}{2}\left(f_{2}{ }^{\prime}+f_{6}{ }^{\prime \prime}\right)
$$

According to geometrical relationship of relative motion once again:

$$
\begin{aligned}
& \Delta h_{2}=\frac{3}{2} \Delta h_{3}-\frac{1}{2} \Delta h_{5} \\
& \Delta h_{6}=\frac{3}{2} \Delta h_{5}-\frac{1}{2} \Delta h_{3}
\end{aligned}
$$

According to the reverse bending deflections and adjustment amounts of $2 \#$ and $6 \#$ roller and the geometrical relationship of relative motion, the adjustment amounts of $1 \#$ and $7 \#$ roller are calculated:

$$
\begin{aligned}
& \Delta h_{1}=\frac{2 t_{1}}{t_{2}}\left(f_{2} "-\Delta h_{2}\right) \\
& \Delta h_{7}=\frac{2 t_{1}}{t_{2}}\left(f_{6} "-\Delta h_{6}\right)
\end{aligned}
$$


Finaly, through arranging these equations above, the adjustment amounts of $1 \#, 2 \#, 4 \#, 6 \#$, and $7 \#$ roller are obtained:

$$
\left.\begin{array}{l}
\Delta h_{1}=\frac{t_{1} t_{2}}{48}\left(\frac{2 \sigma_{\mathrm{s}}}{h E}-3 \xi_{3} \frac{1}{\rho_{3}}\right) \\
\Delta h_{2}=\frac{t_{2}}{8}\left(\frac{2 t_{1}-t_{2}}{3} \frac{1}{\rho_{3}}+\xi_{3} \frac{t_{2}}{4} \frac{1}{\rho_{3}}-\frac{t_{2} \sigma_{\mathrm{s}}}{6 h E}\right) \\
\Delta h_{4}=\frac{t_{2}}{48}\left[\frac{t_{2}}{2}\left(\xi_{3} \frac{1}{\rho_{3}}+\frac{2 \sigma_{\mathrm{s}}}{h E}\right)+\left(2 t_{1}-t_{2}\right)\left(\frac{1}{\rho_{3}}+\frac{2 \sigma_{\mathrm{s}}}{h E}\right)\right] \\
\Delta h_{6}=\frac{t_{2}}{8}\left(\frac{2 t_{1}-t_{2}}{3} \frac{2 \sigma_{\mathrm{s}}}{h E}+\frac{t_{2}}{4} \frac{2 \sigma_{\mathrm{s}}}{h E}-\xi_{3} \frac{t_{2}}{12} \frac{1}{\rho_{3}}\right) \\
\Delta h_{7}=\frac{t_{1} t_{2}}{48}\left(\xi_{3} \frac{1}{\rho_{3}}-\frac{6 \sigma_{\mathrm{s}}}{h E}\right)
\end{array}\right\}
$$

\section{Finite element simulation}

\subsection{Establishment of Finite Element Model}

Using the formula (15), the straightening intermesh schedules of the specifications of plate as shown in Table 1 are calculated, and the finite element models are established in the Abaqus.

Take the $400 \mathrm{MPa} / 50 \mathrm{~mm}$ specification plate as the example to descript the finite element modeling process.

Table 1. Specifications of plate need to be straightening Simulated

\begin{tabular}{|c|c|c|c|c|c|c|}
\hline Specifications & $\begin{array}{c}\text { Spe } \\
\text { c1 }\end{array}$ & $\begin{array}{c}\text { Spe } \\
\text { c2 }\end{array}$ & $\begin{array}{c}\text { Spe } \\
\text { c3 }\end{array}$ & $\begin{array}{c}\text { Spe } \\
\text { c4 }\end{array}$ & $\begin{array}{c}\text { Spe } \\
\text { c5 }\end{array}$ & $\begin{array}{c}\text { Spe } \\
\text { c6 }\end{array}$ \\
\hline $\begin{array}{c}\text { Yield } \\
\text { strength/MPa }\end{array}$ & 400 & 400 & 400 & 300 & 500 & 700 \\
\hline Thickness/mm & 30 & 50 & 70 & 50 & 50 & 50 \\
\hline
\end{tabular}

To improve the computational efficiency, the $1 / 2$ model is adopted, and the work roller is analytical rigid, the whole model is shown in Figure 3. The unevenness of plate is generated randomly; the coordinate distribution along the length direction is shown in Figure 4. The maximum difference of plate in high direction (Y direction) is about $38 \mathrm{~mm}$, and the plate unevenness is 7.3 $\mathrm{mm} \cdot \mathrm{m}^{-1}$. The elastic modulus of steel is $21000 \mathrm{MPa}$, the poison's ratio is 0.3 , and the material stress-strain curve is shown in Figure 5.The plate model is divided by C3D8R (eight nodes linear grid), whose size is $50 \mathrm{~mm} * 25$ $\mathrm{mm} * 5 \mathrm{~mm}, 57200$ elements in total.

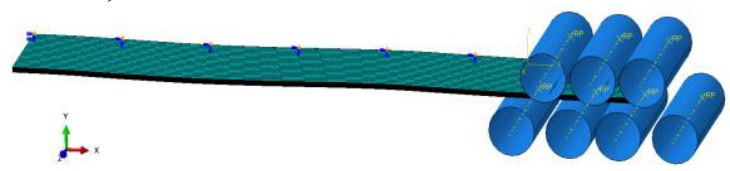

Figure 3. The whole model

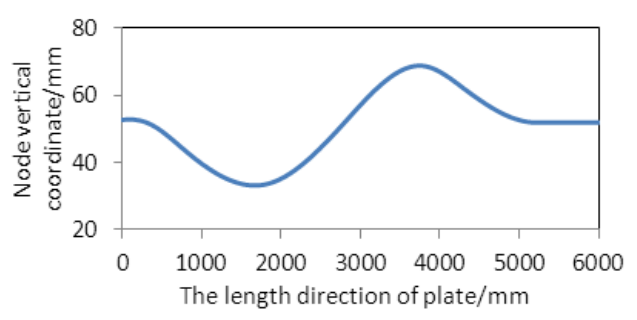

Figure 4. Coordinate distribution of plate intermediate nodes along the length direction before straightening

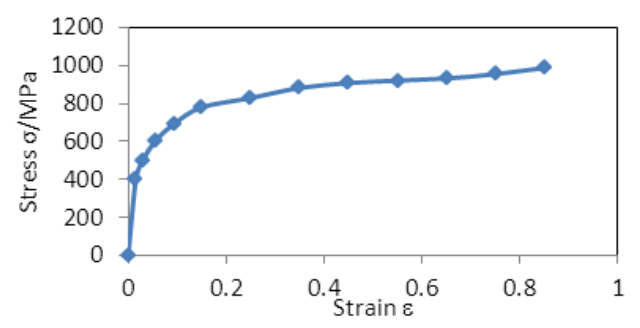

Figure 5. The material stress-strain curve

\subsection{Analysis of Results}

Through simulation, the stress distribution of $400 \mathrm{MPa} / 50$ $\mathrm{mm}$ plate in the roller system is shown in Figure 6. The maximum stress value is $639 \mathrm{MPa}$, and most of the stress value exceeds the yield strength of $400 \mathrm{MPa}$. Therefore, plastic deformation of plate in the straightening simulation process occurs. In addition, due to the work hardening, with reverse bending deformation, although the intermesh amount of last straightening unit is smaller , the stress value is increased.

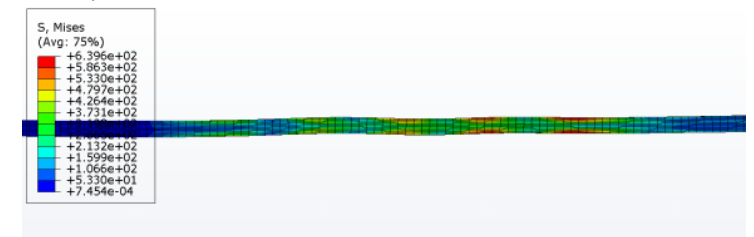

Figure 6. Mises stress distribution of the plate in the roll system

The node coordinates in the middle of the plate after straightening are extracted and rotated according to the coordinate rotation formula (16), and the starting point of the curve is translated to the origin of the coordinate. So the curve is shown in Figure 7.

$$
\left.\begin{array}{l}
x=x_{1} \cos \theta+y_{1} \sin \theta \\
y=-x_{1} \sin \theta+y_{1} \cos \theta
\end{array}\right\}
$$

Where: $(x, y)$ : coordinate after rotation, $\left(x_{1}, y_{1}\right)$ : coordinate before rotation, $\theta$ :the angle between original graph and rotation graph.

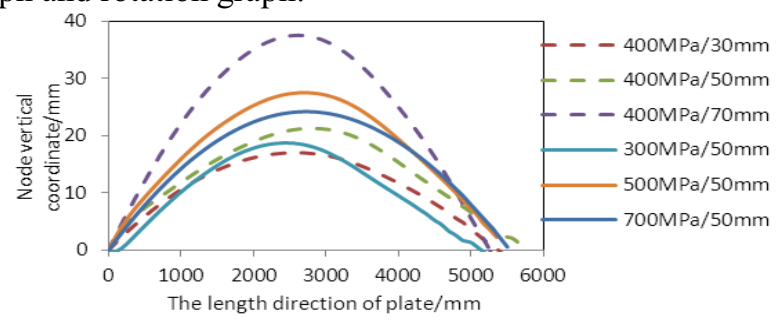

Figure 7. Coordinate value curve of plate nodes after straightening 
As shown in the figure, the unevenness of the plates after straightening get some improvement, but cannot meet the flatness requirements of high quality plate (less than $\left.1 \mathrm{~mm} \cdot \mathrm{m}^{-1}\right)$. In addition it can be found from the figure that along the length of the plate the two bends turn into one, and the residual curvature tends to be consistent. Through analysis and inference, the reason for this phenomenon is that after the plastic deformation in front of the straightening unit the steel plate produces work hardening and the yield strength of the material increases, while the yield curvature increases, but the intermesh amount in the simulation is calculated by the original yield strength, so the anti bending deflection of the last one straightening unit is too small, namely the intermesh of $6 \#$ roller is not enough . In this case, the modified coefficient $X$ is introduced to improve this phenomenon. The anti bending deflection of $6 \#$ roller is:

$$
f_{6}^{\prime \prime}=\mathrm{X} \frac{t_{2}}{24}\left(2 t_{1}-t_{2}\right) \frac{1}{\rho_{6}}
$$

After modifying, the calculation formula of intermesh schedule is:

$$
\begin{aligned}
\Delta h_{1} & =\frac{t_{1} t_{2}}{48}\left(\frac{2 \sigma_{\mathrm{s}}}{h E}-3 \xi_{3} \frac{1}{\rho_{3}}\right) \\
\Delta h_{2} & =\frac{t_{2}}{8}\left(\frac{2 t_{1}-t_{2}}{3} \frac{1}{\rho_{3}}+\xi_{3} \frac{t_{2}}{4} \frac{1}{\rho_{3}}-\frac{t_{2} \sigma_{\mathrm{s}}}{6 h E}\right) \\
\Delta h_{4} & =\frac{t_{2}}{48}\left[\frac{t_{2}}{2}\left(\xi_{3} \frac{1}{\rho_{3}}+\frac{2 \sigma_{\mathrm{s}}}{h E}\right)+\left(2 t_{1}-t_{2}\right)\left(\frac{1}{\rho_{3}}+\mathrm{X} \frac{2 \sigma_{\mathrm{s}}}{h E}\right)\right] \\
\Delta h_{6} & =\frac{t_{2}}{8}\left(\mathrm{X} \frac{2 t_{1}-t_{2}}{3} \frac{2 \sigma_{\mathrm{s}}}{h E}+\frac{t_{2}}{4} \frac{2 \sigma_{\mathrm{s}}}{h E}-\xi_{3} \frac{t_{2}}{12} \frac{1}{\rho_{3}}\right) \\
\Delta h_{7} & =\frac{t_{1} t_{2}}{48}\left(\xi_{3} \frac{1}{\rho_{3}}-\frac{6 \sigma_{\mathrm{s}}}{h E}\right)
\end{aligned}
$$

\subsection{Determine the Modified Coefficient}

Adjusting the modified coefficient, the intermesh schedules corresponding different modified coefficients for each specifications of plates are calculated. Through simulating respectively, then extracting and processing the result data, the modified coefficients corresponding straightening effect (the unevenness is less than $1 \mathrm{~mm} \cdot \mathrm{m}^{-}$ ${ }^{1}$ ), as shown in Figure 8., meeting the flatness requirement is determined, as shown in the Table 2.

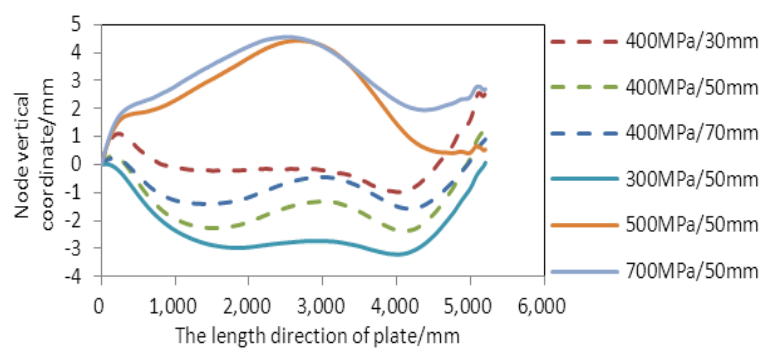

Figure 8. Coordinate value curves of $400 \mathrm{MPa}$ with different thickness plate after straightening
Table 2. Modified coefficients corresponding to different specification of plates

\begin{tabular}{|c|c|c|c|c|c|c|}
\hline Specifications & $\begin{array}{c}\text { Spec } \\
\mathbf{1}\end{array}$ & $\begin{array}{c}\text { Spec } \\
\mathbf{2}\end{array}$ & $\begin{array}{c}\text { Spec } \\
\mathbf{3}\end{array}$ & $\begin{array}{c}\text { Spec } \\
\mathbf{4}\end{array}$ & $\begin{array}{c}\text { Spec } \\
\mathbf{5}\end{array}$ & $\begin{array}{c}\text { Spec } \\
\mathbf{6}\end{array}$ \\
\hline $\begin{array}{c}\text { Yield } \\
\text { strength/MPa }\end{array}$ & 400 & 400 & 400 & 300 & 500 & 700 \\
\hline Thickness/mm & 30 & 50 & 70 & 50 & 50 & 50 \\
\hline $\begin{array}{c}\text { modified } \\
\text { coefficient }\end{array}$ & 1.13 & 1.45 & 1.90 & 1.46 & 1.42 & 1.43 \\
\hline
\end{tabular}

From the table, it can be found that the thickness of the plate has a great influence on the modified coefficient in the formula, and the value of the modified coefficient increases with the increase of the thickness. Through analysis, the reason is that the work hardening of plate after anti bending deformations in the first four straightening units increases with the increasing of thickness, and the insufficient degree of intermesh amount of $6 \#$ roller also increases, so the modified coefficient is larger. On the other hand, the yield strength of the plate has a small influence on the modified coefficient. This is because that the insufficient of intermesh amount of $6 \#$ roller is related to the degree of variation of yield strength of the material before and after work hardening, but is not directly related to the size of the yield strength before work hardening.

In order to apply modified coefficient to practice more conveniently for the plates with different thickness and different yield strength, the modified coefficient $X$ is summarized as the product of thickness effect coefficient $X_{h}$ and strength effect coefficient $X_{\sigma}$, namely $X=X_{h} X_{\sigma}$ 。 Because the influence of plate yields strength on the modified coefficient is small, on the basis of the modified coefficient of $400 \mathrm{MPa} / 50 \mathrm{~mm}$ plate, each $50 \mathrm{~mm}$ thickness plate corresponding to the correction coefficient in the Table 2. is divided by 1.45 that is modified coefficient of $400 \mathrm{MPa} / 50 \mathrm{~mm}$ plate, and the values of strength effect coefficient are obtained, as shown in Table 3. And the value of the plate thickness effect coefficient is the modified coefficient of different thickness plate with $400 \mathrm{MPa}$ yield strength, that is the modified coefficients corresponding to Spec $1-$ Spec 3 in the Table 2.

Table 3. The values of strength effect coefficient

\begin{tabular}{|l|l|l|l|l|l|}
\hline Yield strength /MPa & 300 & 400 & 500 & 600 & 700 \\
\hline Strength effect coefficient & 1.034 & 1.000 & 0.979 & 0.986 & 0.986 \\
\hline
\end{tabular}

The thickness effect coefficient and the strength effect coefficient are anchored point in the respective coordinate system and the value curves are fitted then, as shown in Figure 9 and Figure 10. The two curves can be used to read value for a certain specification plate in practice, and the modified coefficient $X$ is calculated, then the values are taken into the formula (18), and the intermesh schedule is obtained finally. 


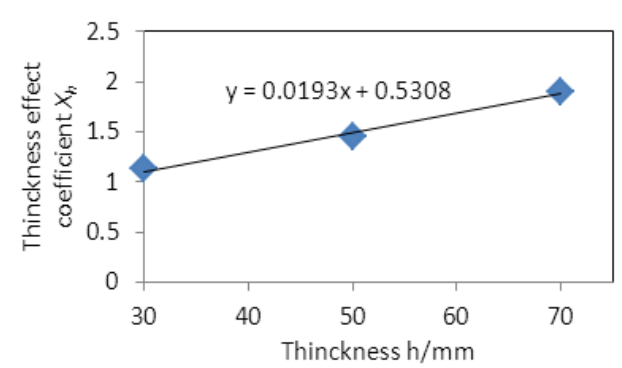

Figure 9. The value curve of thickness effect coefficient

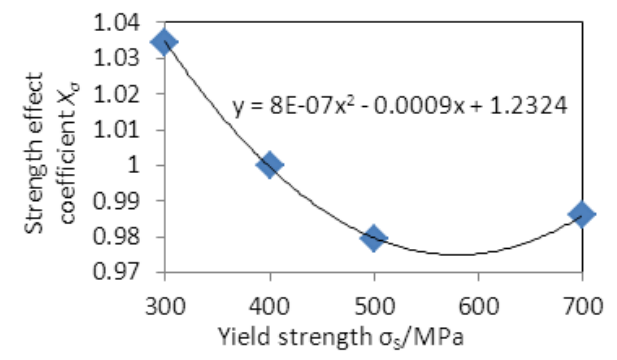

Figure 10. The value curve of strength effect coefficient

\section{Verification}

In order to verify whether the straightening quality of the calculation formula of intermesh schedule after modifing is improved, the straightening intermesh schedules of plates of $400 \mathrm{MPa} / 40 \mathrm{~mm}, 400 \mathrm{MPa} / 60 \mathrm{~mm}$ and 600 $\mathrm{MPa} / 50 \mathrm{~mm}$ specifications are calculated by the modified formula, then the finite element models are established and simulated. The result dates are processed to observe whether the plates after straightening meet the flatness requirement.

According to the thickness and yield strength of the plates need to be verified, thickness effect coefficient and strength effect coefficient are obtained from the figure 9 and 10. The modified coefficient is the product of thickness effect coefficient and strength effect coefficient, and then the modified coefficient is brought into the formula (18), the intermesh amount of each roller is calculated. The finite element models of straightening process are established and simulated, and then plate node coordinates of simulation results are extracted and processed according to the above method. The unevennesses of the plates verified after straightening are obtained and shown in the table 4 . From the table, it is found that each unevenness of the plates after straightening is less than $1 \mathrm{~mm} \cdot \mathrm{m}^{-1}$, and meets the flatness requirement of high quality plate. Therefore, the modified calculation formula of straightening intermesh schedule meets the accuracy requirements, and can be used in formulating the straightening ingtermesh schedule for the newly developed 2800 seven-roller strong heavy plate straightening machine in the practical production.

Table 4. The values of modified coefficient of the plates need to be verified and the unevenness after straightening

\begin{tabular}{|c|c|c|c|}
\hline $\begin{array}{c}\text { Plate } \\
\text { specifications }\end{array}$ & $\begin{array}{c}400 \mathrm{MPa} \\
/ 40 \mathrm{~mm}\end{array}$ & $\begin{array}{c}400 \mathrm{MPa} \\
/ 60 \mathrm{~mm}\end{array}$ & $\begin{array}{l}600 \mathrm{MPa} \\
150 \mathrm{~mm} \\
\end{array}$ \\
\hline Thickness effect & 1.31 & 1.68 & 1.45 \\
\hline
\end{tabular}

\begin{tabular}{|c|c|c|c|}
\hline coefficient Xh & & & \\
\hline $\begin{array}{l}\text { Strength effect } \\
\text { coefficient } \mathrm{X} \sigma\end{array}$ & 1 & 1 & 0.98 \\
\hline $\begin{array}{c}\text { Modified } \\
\text { coefficient X }\end{array}$ & 1.31 & 1.68 & 1.42 \\
\hline $\begin{array}{c}\text { Unevenness/ } \\
\mathrm{mm} \cdot \mathrm{m}-1\end{array}$ & 0.65 & 0.77 & 0.56 \\
\hline
\end{tabular}

\section{Conclusions}

Regarding straightening unit as the research object, considered the different of roller spacing, a mathe-matical model of intermesh schedule suitable to the 2800 sevenroller strong heavy plate straightening machine is deduced by the geometric method, and a new calculationg method of intermesh schedule is offered for other heavy plate straightening machine.

According to mathematical model, the intermesh schedule is calculated, and the finite element model of straightening process is established in the FEA software Abaqus . Through simulation, it is found that the residual curvature decreases and tends to be consistent, but has not yet reached the flatness re-quirements . By analyzing, the reason is that because of the existence of work harding, the anti bending deflection of the last straightening unit is too small.

Introducing modified coefficient $X$, a new calculation formula of straightening intermesh schedule is obtained, and the value of the modified coefficient is obtained by the finite element method.

Through the verification of other specification plates, the modified calculation formula of straightening intermesh schedule meets the accuracy requirements, and together with the value curves of the effect coefficient, can provide theoretical basis for the development of the new heavy plate straightening machine and the formulation of straightening intermesh schedule.

\section{References}

1. W. Zhigang, W. Hui, L. Yourong . J.W.U.S.T., Method for Calculating the Intermesh of Straightener and Its Influence on Straightening Quality. 32(4), 347-350 (2009).

2. W. Xiaogang, H. Qingxue, M. Qin, et al. J.P.E., Research of Deficient Deformation Level ing Model in Heavy Plate. 15(5), 191-194 (2008).

3. L. Jiachuang. C.M.P., Straightening Theory and Curling Theory . 1-18 (2011).

4. L. Jiachuang. M.E., Draft Calculation and Setting for Roller Leveller of Strip and Plate.182, 1-4 (2010).

5. H. Yuhua, X. Youzhong, Y. Huilin. H.M., New Exploration of Straightening Deformation Theory on the Roller-strainghtener. 6, 28-30 (1999).

6. L. Yilun. J.C.S.U., A Math Model on Bending Correction and Its Solution. 19(3):316-324(1988).

7. B. Xiangzhong. S.P.,Mechanics of Materials. 159-161 (2007).

8. C. Pu. M.I.P., Straightening Principle and Straightening Machine. 95-98 (2002). 
9. G. Ben, Z. Yong, Q. Weizhuang, et al. J.M.E., Stress Evolvement and Its Influence on Bending Behavior during Roller Leveling Process. 48(2), 81-86 (2012).

10. W. Xiaogang, H. Qingxue, H. Ying. C.M.E.,Correction and Application of Numerical Model on Plate Roller Levelling. 03: 335-338 (2012).

11. M.Fang. J.A.,Complements of Tableaux and Straightening Bideterminants. 322,1170-1186(2009).

12. L.A.De Bkjar, P.F.R.A. M.M.,Integrating Mathematical CAD and Artificial Simulation of Plastic Rotation in Heat-straightening Processes. Appl.,17, 34-40 (1993).

13. Z. Cunlong, W. Guodong, L. Xianghua ,et al. J.P.E., Work Hardening Modulus and Reverse Bend Curvature in Plate Hot Roller Leveling. 14(4), 133135 (2007).

14. G. Benyue, W. Zhigang, L. Yourong, et al. J.P.E., Finite Element Analysis for the Residual Stress of Plate During Straightening Process., 17(6), 116-120 (2010).

15. Z. Huajian, D. Lei. E.I.P., ABAQUS Basics and Cases Master. 28-163 (2012). 